\title{
Fuzzy logic control of vehicle suspensions with dry friction nonlinearity
}

\author{
L EMIR SAKMAN $^{1}$, RAHMI GUCLU ${ }^{2}$ and NURKAN YAGIZ ${ }^{1}$ \\ ${ }^{1}$ Department of Mechanical Engineering, Istanbul University, 34320 Avcilar, \\ Istanbul, Turkey \\ ${ }^{2}$ Department of Mechanical Engineering, Yildiz Technical University, Besiktas, \\ Istanbul, Turkey \\ e-mail: guclu@yildiz.edu.tr
}

MS received 8 November 2004; revised 23 February 2005

\begin{abstract}
We design and investigate the performance of fuzzy logic-controlled (FLC) active suspensions on a nonlinear vehicle model with four degrees of freedom, without causing any degeneration in suspension working limits. Force actuators were mounted parallel to the suspensions. In this new approach, linear combinations of the vertical velocities of the suspension ends and accelerations of the points of connection of the suspension to the body have been used as input variables. The study clearly demonstrates the effectiveness of the fuzzy logic controller for active suspension systems. Suspension working space degeneration is the most important problem in various applications. Decreasing the amplitudes of vehicle body vibrations improves ride comfort. Body bounce and pitch motion of the vehicle are presented both in time domain when travelling over a ramp-step road profile and in frequency domain. The results are compared with those of uncontrolled systems. At the end of this study, the performance and the advantage of the suggested approach and the improvement in ride comfort are discussed.
\end{abstract}

Keywords. Fuzzy logic control; active vehicle suspension; suspension space.

\section{Introduction}

The main function of vehicle suspension systems is to provide effective isolation from roadsurface unevenness, stability and directional control during handling maneuvers, without loss of ride comfort and vehicle support. Traditional vehicle suspension systems are composed of two parallel components which are springs and dampers. Suspension system designers are faced with the problem of determining suspension spring and damper coefficients. Two important factors conflicting with each other must be compromised. These are ride comfort and road holding. Good ride comfort needs soft springs, which however means weak road holding. Further, while designing passive suspensions there is no way to get rid of resonance frequencies such as the most effective one around $1 \mathrm{~Hz}$, which is the result of vehicle

A list of symbols is given at the end of the paper. 
body dynamics. Replacement of spring damper suspensions of automobiles by active systems has the potential to improve ride comfort. A good vehicle suspension system has to reduce the sprung-mass displacement together with acceleration and provide adequate suspension deflection to maintain tyre contact. This helps to improve ride comfort and vehicle maneuverability. Therefore, improvement of the vehicle suspensions systems has gained large interest and has been the subject of greater research and development in recent years. The reasons are commercial as well as scientific. The main aim of the commercial activity is the desire of automotive manufacturers to improve the performance and quality of their products. On the other hand, researchers and control system designers have claimed that active control of the vehicle suspension system is possible when the developments in actuators, sensors and electronics are considered. In the last twenty years, many studies have been published on active and semi-active suspension systems. Prokop \& Sharp (1992) study active automotive suspensions by road preview on a quarter car model. Nonlinear control of a quarter car active suspension is reviewed by Alleyne \& Hedrick (1995). Burton et al (1995) describe the analysis of active and passive quarter-car systems and a full-scale test rig in their paper. Yu \& Crolla (1998) present an optimal self-tuning control algorithm using a quarter model considering both external and internal disturbances. Yagiz \& Yuksek (2001) apply sliding mode control on a full vehicle model.

Dry friction on dampers is one of the main factors affecting ride comfort. On good road surfaces and at low vehicle speeds, the effect of the road input cannot overcome dry friction force and, therefore, the suspensions almost get locked, a condition known as the "boulevard jerk," and an uncomfortable vibration mode sets in due to reduced degrees of freedom (Silvester 1966).

Owing to the continuously changing elevation of the road surface, the classical approach of control algorithms degenerates the suspension gap, preventing the suspensions and controllers from functioning and causes a very harsh and bumpy ride. The aim of this study is to solve this problem with a fuzzy logic controller. Fuzzy logic has come a long way since it was first presented by Zadeh (1965). Since that time, the subject has been the focus of much research. The attention currently being focussed on fuzzy logic is most probably that the result of present popular consumer products employ fuzzy logic and also the availability of FLC processors (Ross 1995). The superiority of this method is based on its simplicity and satisfactory performance. Yoshimura et al (1997) designed an active suspension system for motor coaches, using a skyhook damper and fuzzy logic control. The active control is assumed to be the sum of the effect of the two. Rao \& Prahlad (1997) suggest fuzzy logic-based control for vehicle active suspension. An active suspension system for a quartercar is controlled to reduce the vertical acceleration to a particular level. Kuo \& Li (2000) describe the development of a composite controller for an automobile active suspension system. Simulations are performed on a quarter-car suspension model. Golob (2001) tests and compares several types of decomposed PID fuzzy logic controllers. The proposed decomposed PID FLC is compared with several PID FLCs structures. Nizar et al (2002) design a sliding mode neural network inference fuzzy logic controller to improve ride and comfort. The study is again realized on a quarter car model.

\section{The vehicle model with dry friction nonlinearity}

A vehicle model with dry friction nonlinearity has been presented in figure 1. Controllers have been placed between sprung and unsprung masses, parallel to the suspensions. The vehicle model has four degrees of freedom, which are body bounce $y$, body pitch bounce $\theta$, front 


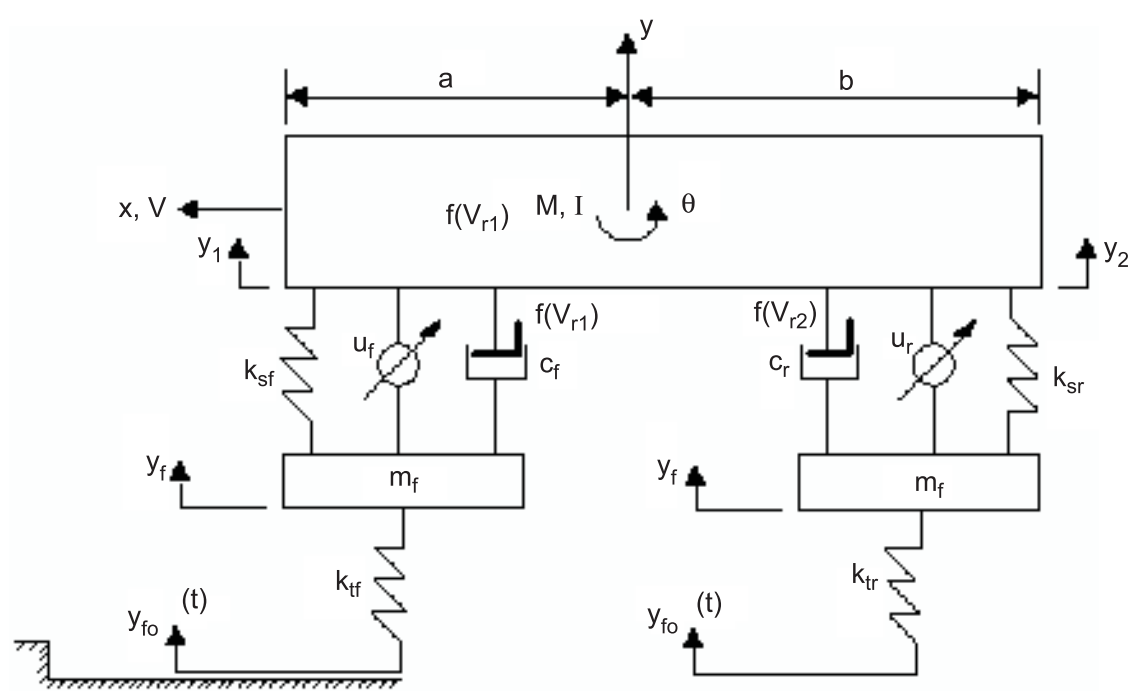

Figure 1. The nonlinear half vehicle model.

wheel hop $y_{f}$ and rear wheel hop $y_{r}$. In this model, $M$ and $I$ represent body mass and inertia, $k_{s f}$ and $k_{s r}$ are front and rear suspension spring constants, $c_{f}$ and $c_{r}$ are front and rear damper coefficients, $f\left(V_{r 1}\right)$ and $f\left(V_{r 2}\right)$ are dry friction forces on front and rear, $u_{f}$ and $u_{r}$ are control force inputs to the front and the rear of the vehicle respectively, $m_{f}$ and $m_{r}$ are front and rear unsprung masses, $k_{t f}$ and $k_{t r}$ are stiffnesses of the front and rear wheels, and $y_{f o}(\mathrm{t})$ and $y_{r o}(\mathrm{t})$ are the front and rear wheel inputs respectively.

The major nonlinearity of the vehicle arises from the dry friction force on dampers. Geometric nonlinearity which arises from the sin and cos terms in deriving the equations of motion has also been included. Dry friction on dampers depends on the relative speed $\left(V_{r}\right)$ between related damper ends. Experiments show that the dry friction model has a viscous band character rather than being of the classical bang-bang type (figure 2). The band $\varepsilon$ is very small, and this prevents the complete locking of the suspension ends. Low speeds on perfect roads generate dry friction force around $\pm R$ that practically locks the suspension, and generates a high equivalent viscous friction effect. Dry friction parameters here are $R=22 \mathrm{~N}$ and $\varepsilon=0.0012 \mathrm{~m} / \mathrm{s}$.

The vehicle travels over the bump in the $x$ direction with velocity, $V . a$ and $b$ are the distances from the body centre to the front and rear suspensions. There is a time delay of $\delta t$

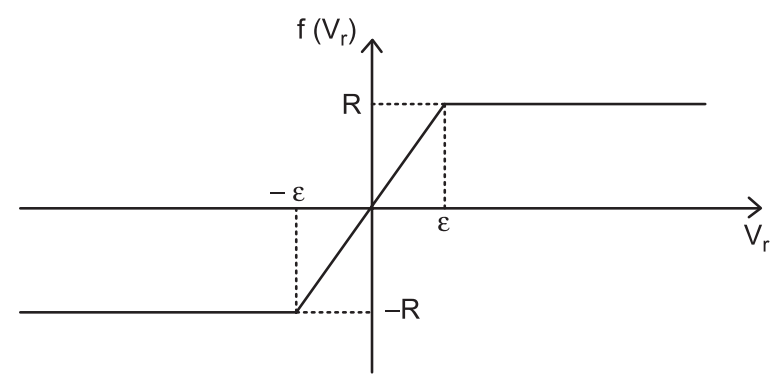

Figure 2. Dry friction model. 
between front and rear wheel road inputs:

$$
\delta t=(a+b) / V .
$$

\section{Fuzzy logic controlled active suspensions}

Negative medium, negative small, zero, positive small and positive medium are used to represent the domain knowledge as linguistic variables. Their membership values lie between 0 and 1. Design of a fuzzy logic controller comprises the following steps:

(i) identifying suitable linguistic variables using the vehicle model parameters (fuzzification);

(ii) setting up a suitable linguistic control rule base;

(iii) setting up suitable logic for control action, based on the measured system variables;

(iv) choosing a method for defuzzification of an interface to scale and mapping the linguistic control actions inferred to yield a non-fuzzy control input to the vehicle being controlled (defuzzification).

The fuzzyfier changes each input variable value to the relevant fuzzy variable value using its own fuzzy sets and their suitable membership functions. Fuzzy sets and their suitable membership functions have to be defined for each output. The defuzzifier is responsible for the translation of the fuzzy reasoning engine results to a crisp set of output values. A variety of methods could be used to perform defuzzification, two of the most common of which are:

i) The Mamdani method that returns the centroid of the output fuzzy region as the crisp output of the fuzzy interface system.

ii) The TVFI (truth value flow inference) method that returns a weighted average as the crisp output of the fuzzy interface system.

In this study the Mamdani method has been used. In the conventional control approach, the aim is to follow zero displacement as a reference value for the body bounce motion. However, when this is realized, it is observed that the suspension working limits degenerate in order to compensate for the difference in the elevation of the vehicle body, in addition to which the amplitude of the road surface displacement changes. This causes the suspensions to malfunction after sometime, and to go out of order, resulting in reduced degrees of freedom and a harsh ride. In order to overcome this practical difficulty a new FLC approach is proposed. The algorithm of the MIMO fuzzy logic controller for the vehicle suspension system uses the errors of the suspension end velocities of the front and rear, and their accelerations and suspension gap velocities as the input variables, while the control forces are $u_{f}$ and $u_{r}$ (Hyniova et al 2001). A model of the two similar rule bases developed by analogy with errors of the front and rear is given in table 1. P, N, ZE, VB, B, M and S represent positive, negative, zero, very big, big, medium and small respectively.

$y_{1}$ and $y_{2}$ are the displacements of the suspension connections to the front and rear of the vehicle. There are 75 rules in the rule base used as presented in table 1, which is divided into two main columns. In the first part, there are 25 rules. In the second part, because of the similarity, 50 rules are defined in 25 rows by using an "or" logical connector. As a result, the 
Table 1. Rule base for the fuzzy logic controllers.

\begin{tabular}{|c|c|c|c|c|c|c|c|}
\hline $\begin{array}{l}e\left(\dot{y}_{1}-\dot{y}_{f}\right) \\
e\left(\dot{y}_{2}-\dot{y}_{r}\right)\end{array}$ & $\begin{array}{l}e\left(\dot{y}_{1}\right) \\
e\left(\dot{y}_{2}\right)\end{array}$ & $\begin{array}{l}e\left(\ddot{y}_{1}\right) \\
e\left(\ddot{y}_{2}\right)\end{array}$ & $\begin{array}{l}u_{f} \\
u_{r}\end{array}$ & $\begin{array}{l}e\left(\dot{y}_{1}-\dot{y}_{f}\right) \\
e\left(\dot{y}_{2}-\dot{y}_{r}\right)\end{array}$ & $\begin{array}{l}e\left(\dot{y}_{1}\right) \\
e\left(\dot{y}_{2}\right)\end{array}$ & $\begin{array}{l}e\left(\ddot{y}_{1}\right) \\
e\left(\ddot{y}_{2}\right)\end{array}$ & $\begin{array}{l}u_{f} \\
u_{r}\end{array}$ \\
\hline PM & PM & ZE & $\mathrm{ZE}$ & PM & PM & $\mathrm{P}$ or $\mathrm{N}$ & PS \\
\hline PS & PM & ZE & PS & PS & PM & $\mathrm{P}$ or $\mathrm{N}$ & PM \\
\hline ZE & PM & $\mathrm{ZE}$ & PM & ZE & PM & $\mathrm{P}$ or $\mathrm{N}$ & PB \\
\hline NS & PM & $\mathrm{ZE}$ & PM & NS & PM & $\mathrm{P}$ or $\mathrm{N}$ & PB \\
\hline NM & PM & $\mathrm{ZE}$ & PB & NM & PM & $\mathrm{P}$ or $\mathrm{N}$ & PVB \\
\hline PM & PS & ZE & ZE & PM & PS & $\mathrm{P}$ or $\mathrm{N}$ & PS \\
\hline PS & PS & $\mathrm{ZE}$ & PS & PS & PS & $\mathrm{P}$ or $\mathrm{N}$ & PM \\
\hline ZE & PS & $\mathrm{ZE}$ & PS & ZE & PS & $\mathrm{P}$ or $\mathrm{N}$ & PM \\
\hline NS & PS & ZE & PM & NS & PS & $\mathrm{P}$ or $\mathrm{N}$ & PB \\
\hline NM & PS & ZE & PM & NM & PS & $\mathrm{P}$ or $\mathrm{N}$ & PB \\
\hline PM & ZE & ZE & NS & PM & ZE & $\mathrm{P}$ or $\mathrm{N}$ & NM \\
\hline PS & ZE & ZE & $\mathrm{ZE}$ & PS & ZE & $\mathrm{P}$ or $\mathrm{N}$ & NS \\
\hline ZE & ZE & ZE & ZE & ZE & ZE & $\mathrm{P}$ or $\mathrm{N}$ & ZE \\
\hline NS & ZE & ZE & ZE & NS & ZE & $\mathrm{P}$ or $\mathrm{N}$ & PS \\
\hline NM & ZE & ZE & PS & NM & ZE & $\mathrm{P}$ or $\mathrm{N}$ & PM \\
\hline PM & NS & ZE & NM & PM & NS & $\mathrm{P}$ or $\mathrm{N}$ & NB \\
\hline PS & NS & $\mathrm{ZE}$ & NM & PS & NS & $\mathrm{P}$ or $\mathrm{N}$ & NB \\
\hline ZE & NS & ZE & NS & ZE & NS & $\mathrm{P}$ or $\mathrm{N}$ & NM \\
\hline NS & NS & ZE & NS & NS & NS & $\mathrm{P}$ or $\mathrm{N}$ & NM \\
\hline NM & NS & $\mathrm{ZE}$ & $\mathrm{ZE}$ & NM & NS & $\mathrm{P}$ or $\mathrm{N}$ & NS \\
\hline PM & NM & $\mathrm{ZE}$ & NB & PM & NM & $\mathrm{P}$ or $\mathrm{N}$ & NVB \\
\hline PS & NM & ZE & NM & PS & NM & $\mathrm{P}$ or $\mathrm{N}$ & NB \\
\hline ZE & NM & $\mathrm{ZE}$ & NM & ZE & NM & $\mathrm{P}$ or $\mathrm{N}$ & NB \\
\hline NS & NM & ZE & NS & NS & NM & $\mathrm{P}$ or $\mathrm{N}$ & NM \\
\hline NM & NM & ZE & ZE & NM & NM & $\mathrm{P}$ or $\mathrm{N}$ & NS \\
\hline
\end{tabular}

rule base used in the simulation is the unique one in table 1 . The rules can be defined using table 1 , beginning from the first row, as

Rule 1:

IF $e\left(\dot{y}_{1}-\dot{y}_{f}\right)$ is PM and $e\left(\dot{y}_{1}\right)$ is PM and $e\left(\ddot{y}_{1}\right)$ is ZE THEN $u_{f}$ is ZE.

Rule 2:

$\mathrm{IF} e\left(\dot{y}_{1}-\dot{y}_{f}\right)$ is PS and $e\left(\dot{y}_{1}\right)$ is PM and $e\left(\ddot{y}_{1}\right)$ is ZE THEN $u_{f}$ is PS.

\section{Rule 26:}

$\mathrm{IF} e\left(\dot{y}_{1}-\dot{y}_{f}\right)$ is PM and $e\left(\dot{y}_{1}\right)$ is PM and $e\left(\ddot{y}_{1}\right)$ is $P$ or $N$ THEN $u_{f}$ is PS.

\section{Rule 27:}

IF $e\left(\dot{y}_{1}-\dot{y}_{f}\right)$ is PS and $e\left(\dot{y}_{1}\right)$ is PM and $e\left(\ddot{y}_{1}\right)$ is $P$ or $N$ THEN $u_{f}$ is PM. 

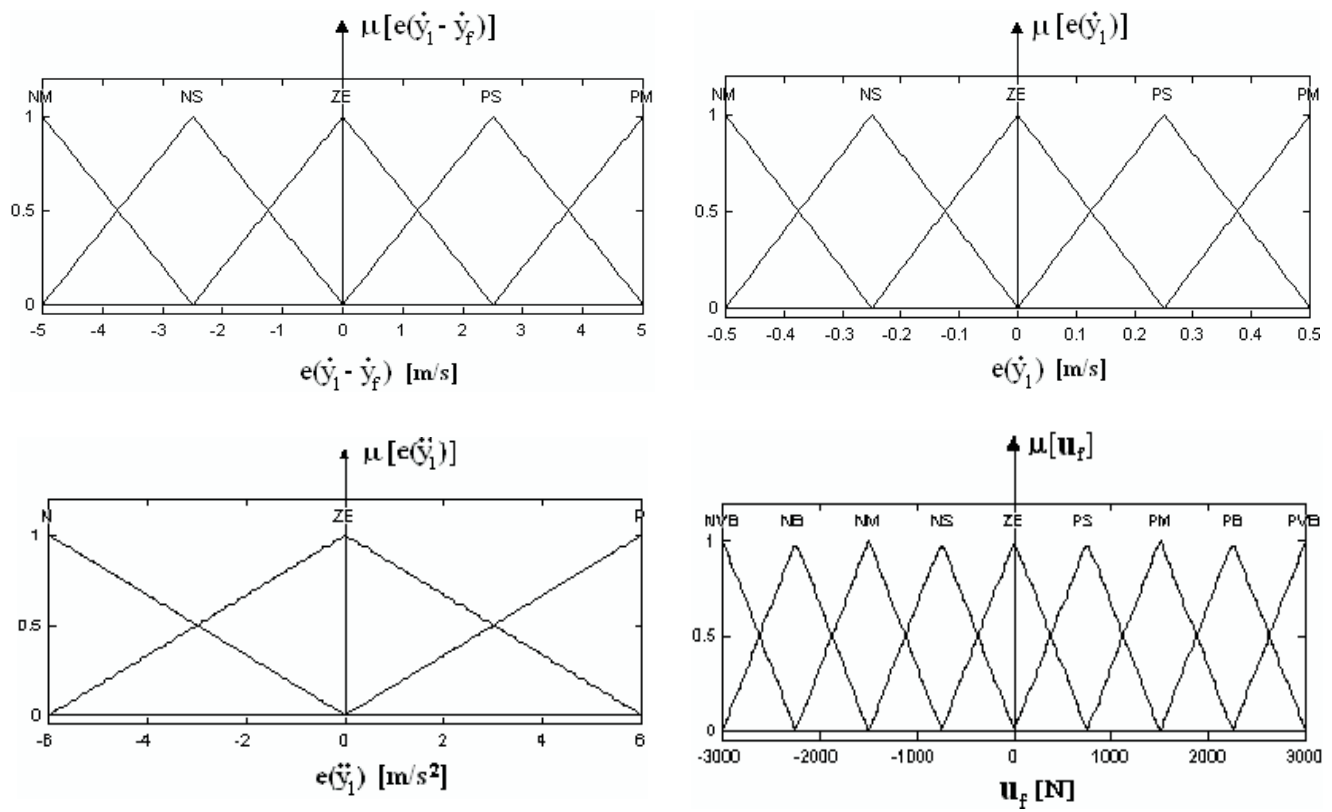

Figure 3. Membership function plots of front body bounce.

The triangular membership functions have been used as shown in figure 3 . The input and output relations in form of Cartesian rule surfaces are presented in figure 4.

\section{Results}

The closed loop model of the fuzzy logic controller for the vehicle suspension system is presented in figure 5. All reference values are assigned as zero.

The study has been carried out using the mathematical model of the system. Road disturbance has been taken as shown in figure 6. It must be considered that there are two road inputs to the system which are applicable to the front wheel and, with a time delay $\delta t$, to the rear wheel. The selection of the road input is very important to check the suspension working
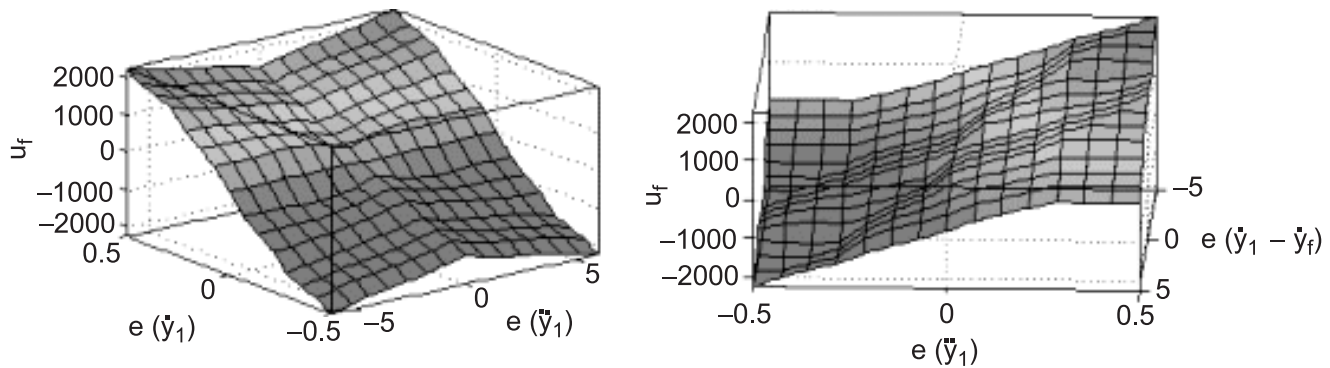

Figure 4. Input and output relations in the form of Cartesian rule surfaces. 


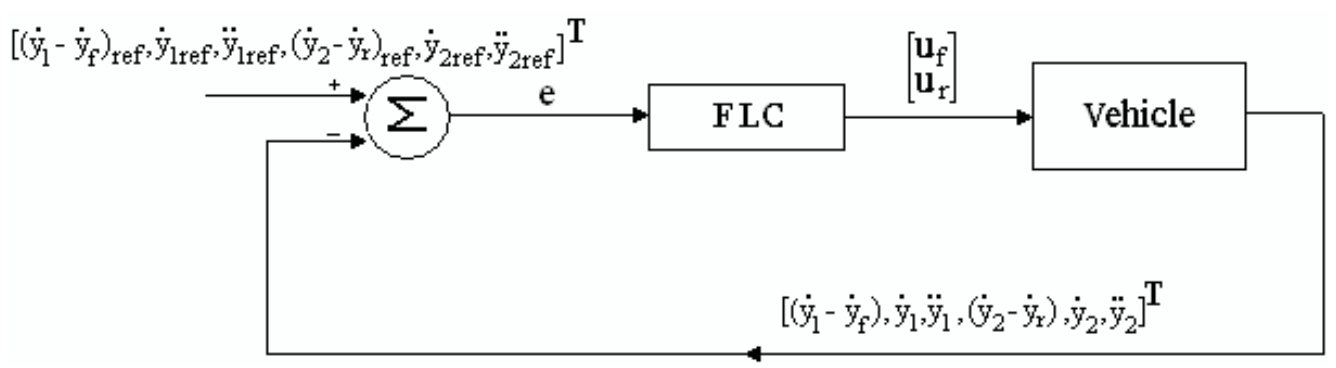

Figure 5. Closed loop model of the vehicle.

space loss problem. If the classical control algorithm were applied, aiming at zero displacement of the vehicle body, there would be a shortening in suspension length in order to realize zero absolute reference change in vehicle body elevation.

Against the test input to the vehicle body, the controlled and uncontrolled vehicle body and pitch displacements and their accelerations are presented in figure 7 . The vehicle body mass follows a smooth trajectory against the road irregularities being sensed by the front and rear wheels as seen in figures $7 \mathrm{a}$ and $\mathrm{b}$. The controlled vehicle body and pitch motion reach zero reference values much faster and are much more comfortable than the uncontrolled ones. Also, the decrease in body bounce acceleration and pitch acceleration values resulting in a more comfortable ride is shown in figures 7c and d. The legend "Controlled" and "Uncontrolled" stand for vehicles with active suspensions and those with passive ones in these figures.

Since the final value of vehicle body displacement equals road surface height, there is no loss in working space of suspensions under control. In fact, changes in suspension lengths are presented in figures $8 \mathrm{a}$ and $\mathrm{b}$. As demonstrated in these figures, there is no permanent change in controlled suspension lengths, which is very important. As seen in these figures, the suspensions reach their original lengths faster and in a smoother manner. The maximum values of the control forces are around $800 \mathrm{~N}$ as seen in figures $8 \mathrm{c}$ and $\mathrm{d}$.

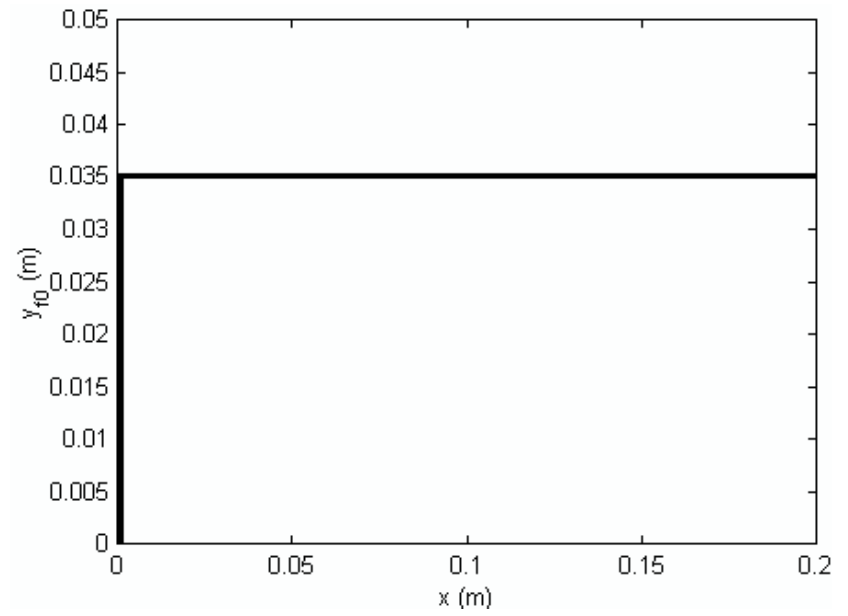

Figure 6. Step road surface input. 

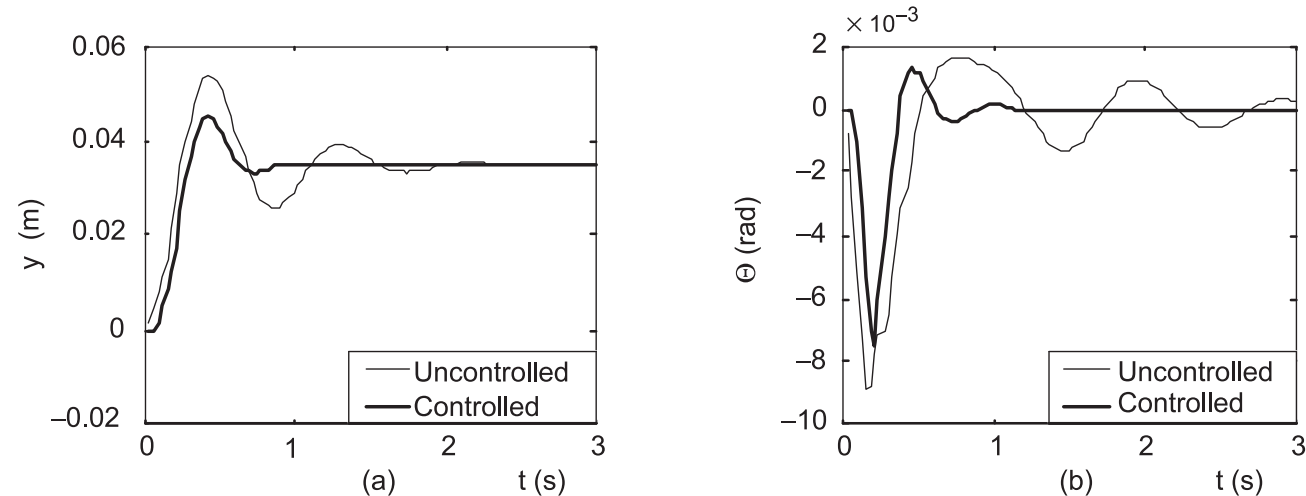

(a)

$\mathrm{t}(\mathrm{s})$

(b)
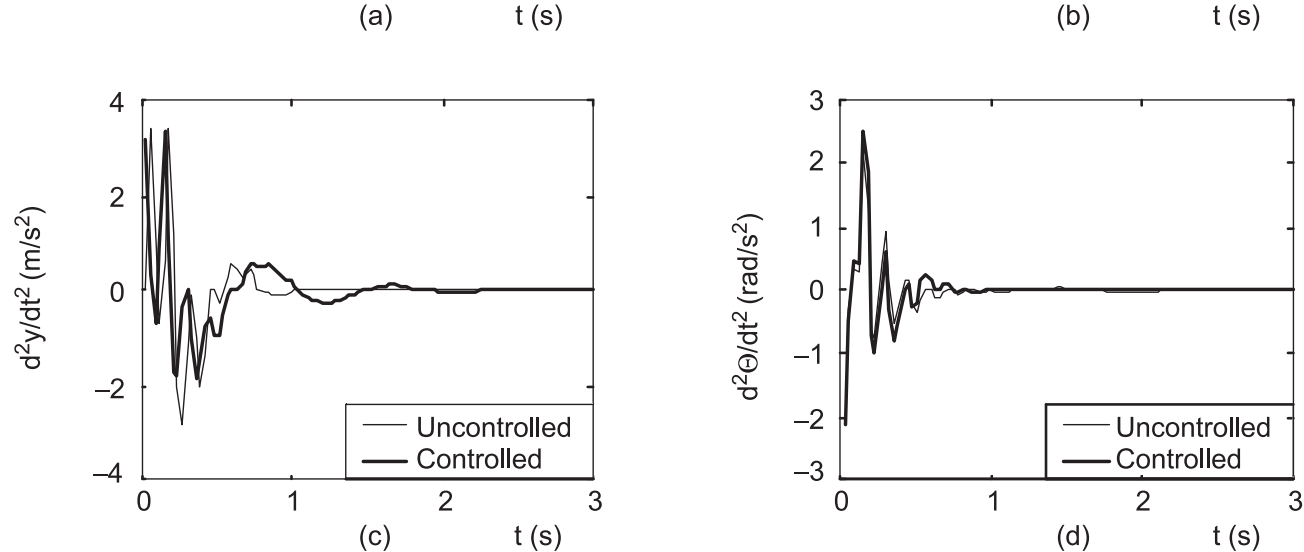

Figure 7. Time response plots of the vehicle body motions; (a) Vehicle body displacement; (b) pitch motion; (c) vehicle body acceleration; (d) pitch acceleration.

After linearization, if the frequency response of the vehicle without controllers is checked, two resonance frequencies are observed around 1.4 and $15 \mathrm{~Hz}$ of body motion and wheel hops as shown in figures $9 \mathrm{a}$ and $\mathrm{b}$. On the other hand, when the controllers are active, the resonances of the body motions belonging to body bounce and pitch disappear and the amplitude of the motion over the most of the frequency range effectively becomes smaller as shown in the same figures. In figures $9 \mathrm{c}$ and d, similar conclusions are obtained if frequency response of accelerations of the body bounce and pitch motion are checked in which the improvement observed is very important when ride comfort is taken into consideration.

In the frequency domain, it is observed that only one mode is controlled. This mode belongs to the vehicle body on which the passengers travel. Besides, since the actuators apply negative control forces on the axles, aiming for control of the vehicle body bounce and pitch causes disturbance of the motion of the unsprung masses. However, this has no negative effect on ride comfort.

\section{Conclusions}

In this study, two fuzzy logic controllers for a nonlinear vehicle have been designed and the results have been presented. The suggested control approach does not cause any degeneration 


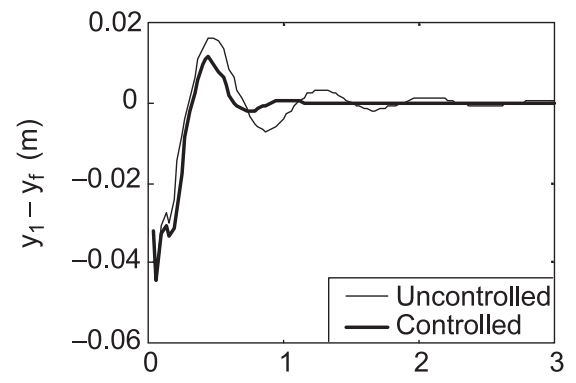

(a)

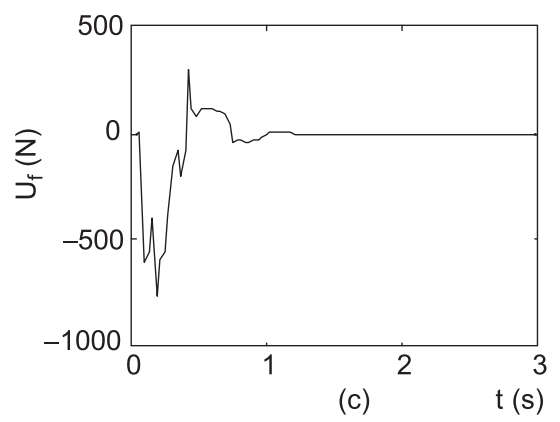

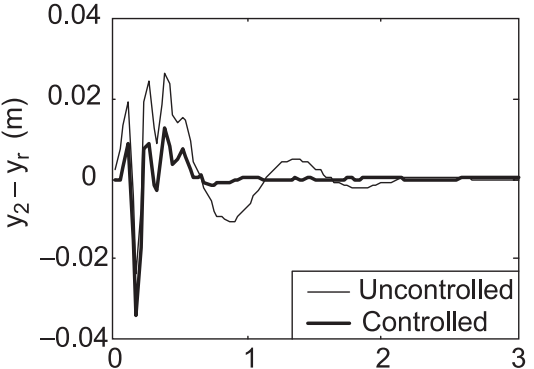

(b)

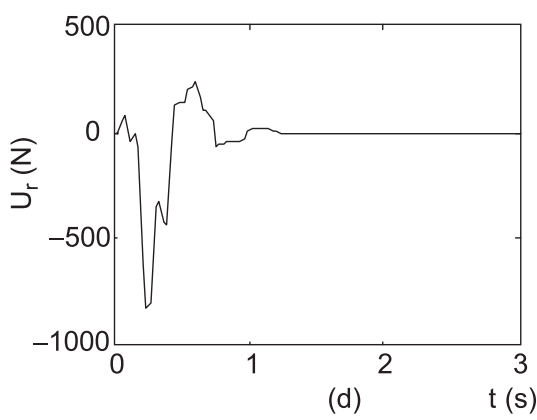

Figure 8. Suspension lengths and forces. (a) Change in front suspension length; (b) change in rear suspension length; (c) front suspension control input force; (d) rear suspension control input force.
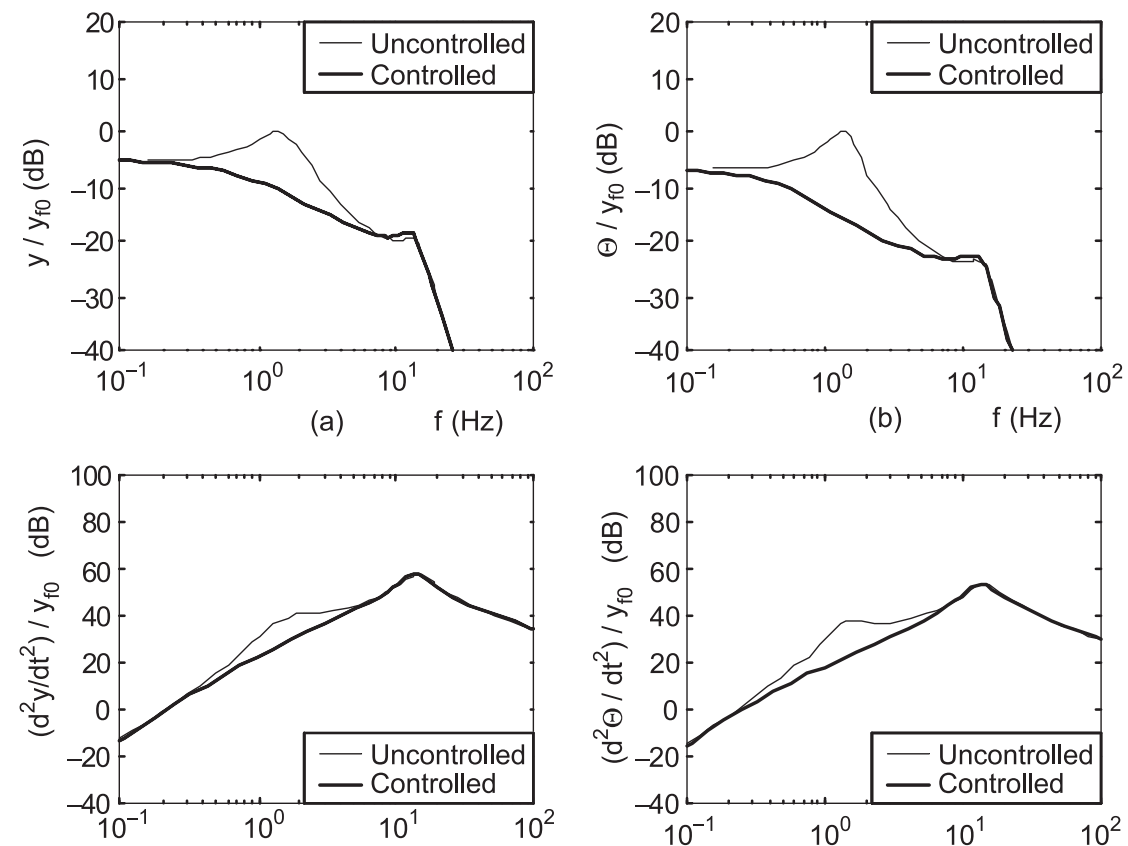

(c) $\quad \mathrm{f}(\mathrm{Hz})$

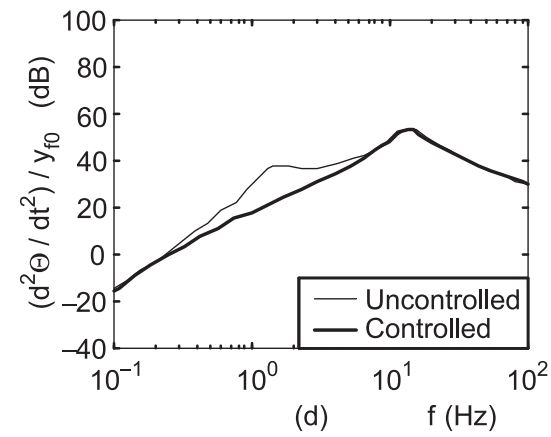

Figure 9. Frequency response plots of the vehicle body motions; (a) Vehicle body displacement; (b) pitch motion; (c) vehicle body acceleration (d) pitch acceleration. 
in suspension working limits, while it improves ride comfort as was the objective of this study. The results prove that the proposed active suspension system is very effective in vibration isolation of the vehicle body. The idea behind proposing this controller was its success and the ability of using these type of controllers on vehicles with the developing technology and the possibility of overcoming the suspension working space loss problem. The results of this study prove that the performance of the active suspension of this type is superior to that of passive ones and the suggested approach solves the suspension degeneration problem which has so far been the main obstacle in applications.

\section{List of symbols}

$a, b \quad$ distances of axle to the centre of the gravity of the vehicle body;

$c_{f} \quad$ damping coefficient of front suspensions;

$c_{r} \quad$ damping coefficient of rear suspensions;

$e(t) \quad$ error;

$f\left(V_{r}\right)$ dry friction force;

$k_{s f} \quad$ spring constant of front suspensions;

$k_{s r} \quad$ spring constant of rear suspensions;

$k_{t f} \quad$ spring constant of front tyres;

$k_{t r} \quad$ spring constant of rear tyres;

$M \quad$ mass of the vehicle body;

I inertia of the vehicle body;

$u(t) \quad$ control signal;

$V \quad$ velocity of the vehicle;

$x_{\text {ref }}$ reference value.

Appendix A.

Table A1. System parameters.

\begin{tabular}{lcc}
\hline Parameters & Numerical value & Unit \\
\hline$M$ & 1000 & $\mathrm{~kg}$ \\
$I$ & 1600 & $\mathrm{kgm}^{2}$ \\
$m_{f}$ & 100 & $\mathrm{~kg}$ \\
$m_{r}$ & 200 & $\mathrm{~kg}$ \\
$c_{f}$ & 2000 & $\mathrm{Ns} / \mathrm{m}$ \\
$c_{r}$ & 2000 & $\mathrm{Ns} / \mathrm{m}$ \\
$k_{s f}$ & 28000 & $\mathrm{~N} / \mathrm{m}$ \\
$k_{s r}$ & 32000 & $\mathrm{~N} / \mathrm{m}$ \\
$k_{t f}$ & 400000 & $\mathrm{~N} / \mathrm{m}$ \\
$k_{t r}$ & 400000 & $\mathrm{~N} / \mathrm{m}$ \\
$a$ & 1 & $\mathrm{~m}$ \\
$b$ & $1 \cdot 2$ & $\mathrm{~m}$ \\
$V$ & 20 & $\mathrm{~m} / \mathrm{s}$ \\
\hline
\end{tabular}


The mathematical model of the vehicle is:

$$
\begin{aligned}
& M \ddot{y}+\left(c_{f}+c_{r}\right) \dot{y}+\left(k_{s f}+k_{s r}\right) y-\left(a c_{f}-b c_{r}\right) \dot{\theta} \cos \theta-\left(a k_{s f}-b k_{s r}\right) \sin \theta \\
& \quad-c_{f} \dot{y}_{f}-k_{s f} y_{f}-c_{r} \dot{y}_{r}-k_{s r} y_{r}+f\left(V_{r 1}\right)+f\left(V_{r 2}\right)=u_{f}+u_{r}, \\
& I \ddot{\theta}+\left(c_{f} a^{2}+c_{r} b^{2}\right) \dot{\theta} \cos ^{2} \theta+\left(k_{s f} a^{2}+k_{s r} b^{2}\right) \cos \theta \sin \theta+\left(c_{r} b-c_{f} a\right) \cos \theta \dot{y} \\
& \quad+\left(k_{s r} b-k_{s f} a\right) y \sin \theta+c_{f} a \cos \theta \dot{y}_{f}+k_{s f} a \sin \theta \dot{y}_{f}-c_{r} b \cos \theta \dot{y}_{r} \\
& \quad-k_{s r} b \sin \theta y_{r}+a f\left(V_{r 1}\right)-b f\left(V_{r 2}\right)=b u_{r}-a u_{f}, \\
& \left.m_{f} \ddot{y}_{f}+c_{f} \dot{y}_{f}+\left(k_{s f}+k_{t f}\right) y_{f}-c_{f} \dot{y}\right]-k_{s f} y+c_{f} a \dot{\theta} \cos \theta+k_{s f} a \sin \theta \\
& \quad-k_{t f} y_{f 0}-f(V r 1)=-u_{f}, \\
& m_{r} \ddot{y}_{r}+c_{r} \dot{y}_{r}+\left(k_{s r}+k_{t r}\right) y_{r}-c_{r} \dot{y}-k_{s r} y-c_{r} b \dot{\theta} \cos \theta-k_{s r} b \sin \theta \\
& \quad-k_{t r} y_{r 0}-f(V r 2)=-u_{r} .
\end{aligned}
$$

\section{References}

Alleyne A, Hedrick J K 1995 Non-linear adaptive control of active suspensions. IEEE Trans. Contr. Syst. Technol. 3: 95-101

Burton A W, Truscott A J, Wellstead P E 1995 Analysis, modeling and control of an advanced automotive self-leveling suspension system. IEE Proc. Contr. Theor. App. 142: 129-139

Golob M 2001 Decomposed fuzzy proportional-integral-derivative controllers. Appl. Soft Comput. 1: 201-214

Hyniova K, Stribrsky A, Honcu J 2001 Fuzzy control of mechanical vibrating systems. Proc. Int. Carpathian Control Conference, Krakow, pp 393-398

Kuo Y P, Li T H S 2000 A composite EP-based fuzzy controller for active suspension system. Int. Journal of Fuzzy Syst. 2: 183-191

Nizar H, Lahdhiri T, Joo D S, Weaver J, Faysal A 2002 Sliding mode neural network inference fuzzy logic control for active suspension systems. IEEE Trans. Fuzzy Syst. 10: 234-246

Prokop G, Sharp R S 1995 Performance enhancement of limited-bandwidth active automotive suspensions by road preview. IEE Proc. Contr. Theor. App. 142: 140-148

Rao M V C, Prahlad V 1997 A tunable fuzzy logic controller for vehicle-active suspension systems. Fuzzy Sets Syst. 185: 11-21

Ross T J 1995 Fuzzy Logic with Engineering Applications, McGraw-Hill Inc

Silvester B C 1966 Vibration reduction in motor cars. Society of Environmental Engineers, 1966 Symposium, London, p. 4

Yagiz N, Yuksek I 2001 Sliding mode control of active suspensions for a full vehicle model. Int. J. Vehicle Design 26: 264-276

Yoshimura T, Isari Y, Li Q, Hino J 1997 Active suspension of motor coaches using skyhook damper and fuzzy logic control. Contr. Eng. Practice 5: 175-184

Yu F, Crolla D A, 1998 An optimal self-tuning controller for an active suspension. Vehicle Syst. Dynam. 29: 51-65

Zadeh L 1965 Fuzzy sets. Inf. Contr. 8: 338-353 